\title{
Diagnosis and treatment of headache probably attributed to cerebral venous sinus thrombosis
}

\begin{abstract}
Cerebral venous sinus thrombosis (CVST) is a rare condition whose most common and sometimes only symptom is headache. Alas, diagnosis and treatment of CVST is often delayed or overlooked because of its high clinical variability. Using guidelines advices in detecting warning signs or symptoms of secondary headaches might ease the diagnosis of CVST.

The article presents the case of a woman who is in treatment for chronic migraine and assessed for secondary headache in a multidisciplinary outpatient headache program. Alert symptoms like sudden worsening headache presentation, along with anamnestic cues, prompted neuroimaging that detected left transverse sinus thrombosis whose onset was difficult to date.
\end{abstract}

Keywords: Primary Headache; Secondary Headache; Chronic Migraine; Cerebral Venous Sinus Thrombosis

Diagnosi e terapia di una cefalea probabilmente derivante da trombosi dei seni venosi cerebrali CMI 2014; 8(4): 97-101

bttp://dx.doi.org/10.7175/cmi.v8i4.966

\section{INTRODUCTION}

Cerebral venous sinus thrombosis (CVST), a rare condition whose incidence is 3-4 cases per 1 million people ( $75 \%$ of cases are women) and up to 7 cases to 1 million among children, is the presence of a blood clot in the dural venous sinuses draining dehoxygenated blood from the brain [1]. The most common and sometimes only symptom of CVST is headache (80-90\% of patients), while visual symptoms, stroke symptoms, seizures and depressed level of consciousness can be present as well [1,2]. The diagnosis may be suspected on the basis of symptoms, for example the combination of headache, signs of raised intracranial pressure, and focal neurological abnormalities $[1,2]$. The diagnosis is confirmed by neuroimaging showing thrombus obstructing the venous sinuses $[1,2,3]$. CVST risk factors include: thrombophilia, chronic inflammatory diseases,

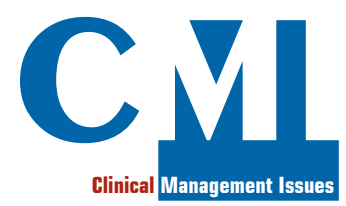

Case report

Centre for Headache and Adaptive Disorders, Unit of Neurology, Pugliese-Ciaccio Hospital, Catanzaro, Italy

${ }^{2}$ Unit of Diagnostic Radiology,

Pugliese-Ciaccio Hospital,

Catanzaro, Italy

${ }^{3}$ Chair of Pharmacology,

School of Medicine and Surgery, Magna Graecia University of Catanzaro, Italy ${ }^{4}$ Chair of Neurosurgery,

School of Medicine and Surgery, Magna Graecia University of Catanzaro, Italy
Why we describe this case

This case provides an example of a situation where is needed to understand that a secondary headache might be in place in a patient with a history of treated chronic migraine, even when acute neuroimaging markers are not detectable anymore. A multidisciplinary headache assessment and management in a hospital outpatient program proved effective in this case
Corresponding author Dr. Rosario Iannacchero Presidio Ospedaliero "Pugliese"

Viale Pio X

88100 Catanzaro, Italy Tel./fax: (+39) 0961883083 centrocefaleeaopc@gmail.com

Disclosure

The authors declare they have no competing financial interests concerning the topics of this article 
pressure, surgical placement of a shunt can be performed. Symptoms like headache are also treated $[4,5,6]$. Alas, the diagnosis and treatment of CVST is often delayed or overlooked because of its high clinical variability [7]. The rate of missed diagnoses and treatment can be reduced using a diagnostic and therapeutic path able to detect symptoms and signs of the CVST presentations and orientate the most appropriate care.

\section{CASE PRESENTATION}

We present the case of M.L., a 35-year-old Italian woman living in Calabria. Following a neurological consult in January 2014, she was referred to the Headache Center at the Pugliese-Ciaccio Hospital in Catanzaro to continue receiving the Botulinum Toxin type A (BTXA) treatment for chronic migraine previously received in a hospital located in another region of Italy. The referral aimed to eliminate the need for long travels to receive therapy.

The patient came to the Headache Center in Catanzaro in March 2014. At a first medical examination, where a headache diary was given to the patient, emerged that the patient was not previously assessed for secondary migraine before or after receiving chronic primary headache treatment. Therefore, $\mathrm{Mrs}$. L. was admitted to a chronic migraine outpatient program aimed to perform a complete headache assessment and subsequent therapeutic prescriptions. The program, that is multidisciplinary (it includes neurological examination, psychological evaluation, disability evaluation, pharmacological assessment, neuroimaging exams, on a need-basis specialist consults and pharmacological and non-pharmacological prescriptions) is designed to be adaptable to the patient presentation, needs, and preferences. As a first step in the program (T1), a medical history assessment, a neurological examination and a psychological evaluation were scheduled and performed in March 2014.

Medical history wise, blood hypertension was present in the familiar medical history in both maternal and paternal sides. Mrs. L. was married, had no kids, holded a university degree in economics and worked as an accountant; she had no history of any substance use or abuse; her personal medical history included: common exanthems in childhood; hyperomocisteinemia treated with daily intake of calcium mefolinate; endometriosis treated with two surgical procedures in 2005; ovarian dysfunction treated with daily oral contraceptives; Mrs. L. was in treatment since 2009 for chronic migraine with BTXA and used diclofenac pills to manage acute episodes of migraine since 1998. Botulinum therapy had initially reduced the frequency of headache days from 25 days/month to 6 days/month.

General physical examination did not show anything to report. Neurological examination showed cranial hypoesthesia; Mrs L. reported suffering of prolonged and repeated fronto-temporal pulsating headache attacks lasting up to one week whose pain intensity increased over the days. Attack treatment with non-steroidal anti-inflammatory drugs was not effective. Reportedly, gait instability and recurrent vomiting had developed. Pain intensity classified by Numeric Rating Scale (NRS) was 8 out of 10 . Headache days had rised to a value of 15 per month.

Psychological evaluation consisted in a clinical interview and the administration of self-report questionnaires to measure anxiety and depression level and to detect pathological anxiety or mood state, the Zung Anxiety Self Report Scale (Zung-Anx) and the Zung Depression Self Report Scale (Zung-Dep). Each of those tests consists of
Figure 1. Left

transverse sinus absence shown by venous Magnetic Resonance Angiography (vMRA)
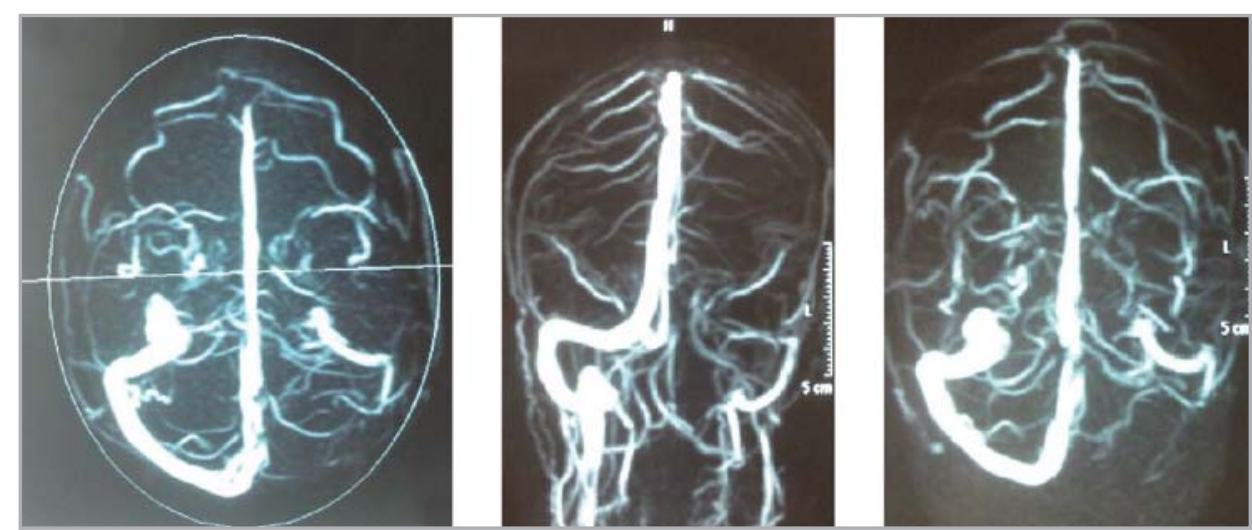
20 affirmations. The subject had to express her degree of agreement on a 4-point scale (from 1 = never to 4 = always). Psychological assessment showed no occurrence of pathological anxiety or mood state, nor any pathological personality trait (Zung-Anx = 33; Zung-Dep = 37). Migraine Disability Assessment (MIDAS) was administered in order to evaluate quality of life impact of the headache in the patient. MIDAS score (108) showed high level of disability experienced by the patient in work, social, and familiar life.

A pharmacological use assessment remarked continuous use of diclofenac tablets $(150 \mathrm{mg})$ as attack treatment since the chronic migraine onset but excluded medications overuse.

Following medical and psychological evaluations, considering the presence of a secondary headache risk factor (usage of oral contraceptives) along with a possible alert symptom (sudden change and refractory worsening of the headache presentation), neuroimaging examinations were prescribed and performed, in order to investigate secondary forms of headache: nuclear Magnetic Resonance Imaging (MRI) and venous Magnetic Resonance Angiography (vMRA).

The MRI showed altered signal around the pineal gland without Fluid-Attenuated Inversion Recovery (FLAIR) signal suppression. The signal was judged possibly indicating a pineal cystis. Also, it showed alteration of the signal of the maxillary sinuses and turbinates of possibly inflammatory nature. The vMRA showed absence of the signal within the left transverse sinus and hypoplasia of sigmoid sinus and gulfs of the ipsilateral jugular (Figure 1).

Considering the vMRA result, a hematological consult to perform a genetic trombophilic investigation was requested and a prothrombin mutation (G20210A) was detected. As a diagnostic conclusion, a secondary headache, headache probably attributed to consequences of cerebral venous thrombosis along with primary chronic migraine, was diagnosed in March 2014 [5]. At the same time, the prescribed therapy was therefore adjusted in light of the recent diagnostic change: thrombolysis and/ or oral anticoagulant were not prescribed to the patient because it was not possible to temporally date the CVST onset; In facts, signal hyper-intensity, acute venous thrombosis marker, was not evident in the MRI

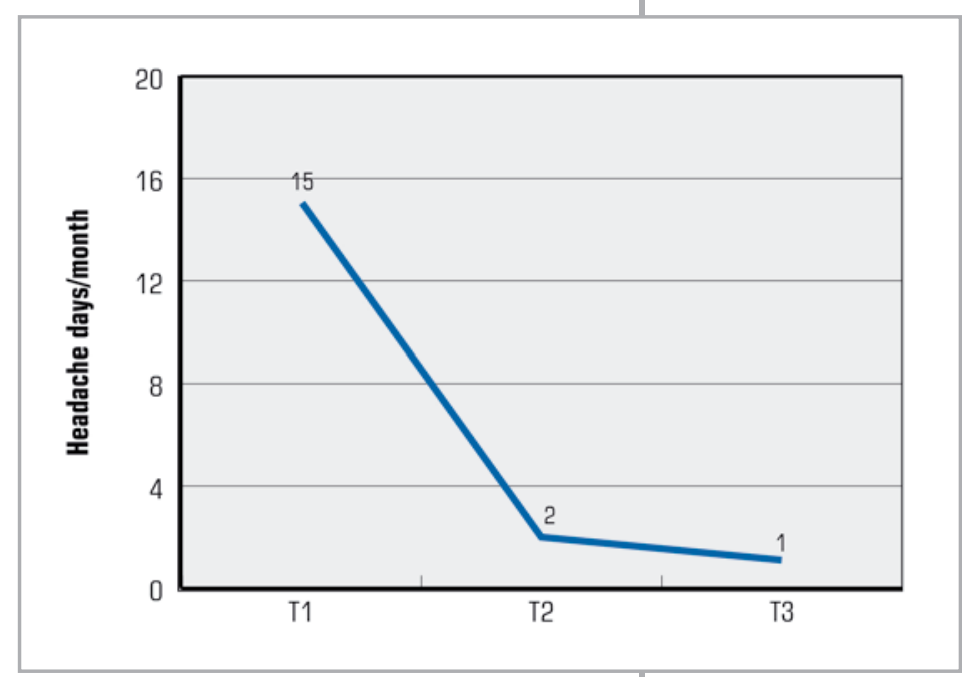

T1 sequence. Therefore, the vMRA finding was judged as a probably old dated thrombosis outcome. After a consultation with the local hospital Hematology and Thrombosis Center, costs-benefits ratio wise, the

Figure 2. Headache frequency in days per month since therapy initiation

$\mathrm{T} 1=$ March 2014

T2 = June 2014

$\mathrm{T} 3=$ September 2014

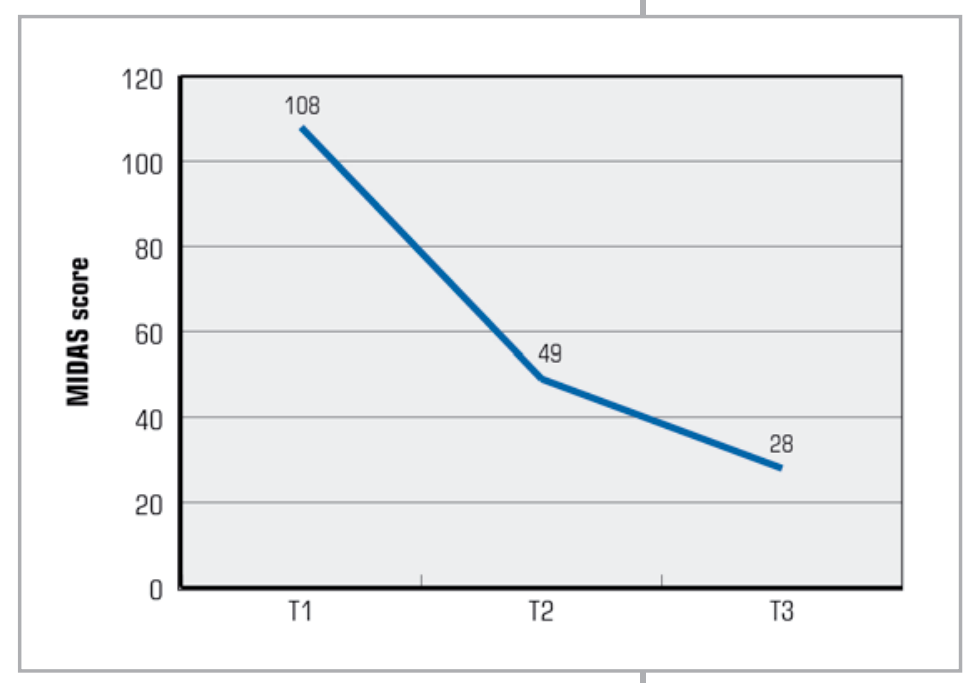

anticoagulant therapy was considered inappropriate, since it was a long but undefined time ago dated thrombosis outcome. Topiramate ( $25 \mathrm{mg} / \mathrm{day}$ ) was prescribed as a prophylactic therapy, while eletriptan (40 $\mathrm{mg}$ ) was used as a once a need treatment. Moreover, the patient continued receiving BTXA (200 units per session) treatment every three months, being the next injection scheduled in April 2014. A follow-up program every three months after each injection session was planned. In June 2014, at the first follow-up examination (T2), the prolonged and pain increasing headache
Figure 3. Degree of disability measured by MIDAS since therapy initiation

$\mathrm{T} 1=$ March 2014

$\mathrm{T} 2$ = June 2014;

$\mathrm{T} 3=$ September 2014 
episodes were reduced to 2 episodes/month lasting one day. Pain intensity $(\mathrm{NRS}=6)$ and degree of disability (MIDAS $=49$ ) were also reduced. In September 2014, at the second follow-up examination (T3), headache frequency had reduced to 1 episode/ month. Pain intensity was higher at onset $(\mathrm{NRS}=8)$ and reduced in duration; degree of disability, while still high (MIDAS $=28$ ), was also reduced (Figure 2, Figure 3). The patient will continue the BTXA treatment and the follow-up program at the Headache Center in Catanzaro. Monitoring time wise, the patient is adviced to undergo a yearly neuroradiological (vMRA) follow-up for 5 years, to monitor the venous obliteration state.

\section{DISCUSSION}

It is often difficult to differentiate between primary and secondary forms of chronic headache, because there are many similarities shared by the various forms and there is a lack of headache-specific diagnostic exams. Thus, differentiate between primary and secondary forms of chronic headache is often matter of application of guidelines in the clinical practice [8]. Secondary chronic headache wise, the primary condition might be unknown and not detectable by the diagnostic tools commonly used. Then, it is important for the clinician to know and detect those medical history cues, signs and symptoms that might be suggestive of a secondary condition and prompt the request for specific examinations. In the case discussed above, a type of primary headache that suddenly became worse to the point of seeming a different type of headache raised concerns that a secondary headache might have been in place. Neuroimaging findings validated that hypothesis by detecting a cerebral venous sinus thrombosis whose onset was, nonetheless, difficult to temporally date. A genetic investigation made sure about the presence of a thrombophilic mutation anyways. Remission of the new type of headache by CVST appropriate treatment might confirm the diagnosis of headache attributed to cerebral venous thrombosis along with a pre-existing and treated primary chronic migraine.

Given that there are no headache-specific diagnostic tools aside of the clinical method, it is essential to conform one's clinical work to the most appropriate headache management guidelines and assess carefully medical history, headache presentation and its changes, medications usage: that will prompt the examination needed to specify the headache form and its possible primary condition.

The patient admission to a comprehensive and multidisciplinary outpatient headache program whose practices adhere to the most updated headache care guidelines, made easier for the clinicians to detect the secondary form of headache, its primary cause and promptly adjust the planned treatment.

\section{When to suspect a secondary headache?}

Certain warning signs or symptoms may suggest a secondary headache, prompting additional examination (blood tests, brain scans, lumbar puncture) and/or referral to a specialist. These include:

- neurological symptoms during or between headaches;

- positive neurological examination;

- new or unusual type of headache;

- sudden or worst headache of one's life;

- headaches unresponsive to treatment and/or steadily worsening;

- older age;

- immunocompromission;

- fever.

In the presented case, a sudden and refractory change in headache presentation, neurological signs along with a continuous contraceptive usage in medical history as a risk factor, prompted magnetic resonance and venous magnetic resonance angiography as additional examinations: neuroimaging detected cerebral venous sinus thrombosis as probably the underlying cause of a secondary headache. Nonetheless, there are no visible previous acute neuroimaging markers for a thrombosis that has probably happened a significant time before the examination. 


\section{REFERENCES}

1. Stam J. Thrombosis of the cerebral veins and sinuses. N Engl J Med 2005; 352: 1791-8; http:// dx.doi.org/10.1056/NEJMra042354

2. Cumurciuc R, Crassard I, Sarov M, et al. Headache as the only neurological sign of cerebral venous thrombosis: a series of 17 cases. J Neuro Neurosurg Psychiatr 2005; 76: 1084-7; http:// dx.doi.org/10.1136/jnnp.2004.056275

3. Smith R, Hourihan MD. Investigating suspected cerebral venous thrombosis. BMJ 2007; 334: 794-5; http://dx.doi.org/10.1136/bmj.39154.636968.47

4. Einhäupl K, Bousser MG, de Bruijn SF, et al. EFNS guideline on the treatment of cerebral venous and sinus thrombosis. Eur J Neurol 2006; 13: 553-9; http://dx.doi.org/10.1111/j.14681331.2006.01398.x

5. Stam J, De Bruijn SF, DeVeber G. Anticoagulation for cerebral sinus thrombosis. Cochrane Database Syst Rev 2002; (4): CD002005; http://dx.doi.org/10.1002/14651858.CD002005

6. Martinelli I, Franchini M, Mannucci PM. How I treat rare venous thromboses. Blood 2008; 112: 4818-23; http://dx.doi.org/10.1182/blood-2008-07-165969

7. Chiewvit P, Piyapittayanan S, Poungvarin N. Cerebral venous thrombosis: diagnosis dilemma. Neurol Int 2011; 3: e13; http://dx.doi.org/10.4081/ni.2011.e13

8. International Headache Society, The International Classification of Headache Disorders, 3rd edition (beta version). Cephalalgia 2013; 33: 629-808 